\title{
Management of sentinel lymph nodes in lower extremity melanoma with dual-basin (inguinal and popliteal) drainage on lymphoscintigraphy
}

\author{
Joonhyuk Son, Jung-Han Kim, Ji Young Seong, Nayoon Hur, Jun-Ho Choe, Jee Soo Kim \\ Department of Surgery, Samsung Medical Center, Sungkyunkwan University School of Medicine, Seoul, Korea
}

Purpose: To analyze clinical outcomes of sentinel lymph node (SLN) biopsy in lower extremity melanoma with dual-basin drainage on lymphoscintigraphy.

Methods: We retrospectively analyzed patients with lower extremity melanoma who underwent lymphoscintigraphy and SLN biopsy in a single center between 2006 and 2015. Gamma probe was used to detect SLNs. For dual-basin drainage, we regarded a basin that showed a two-fold greater radioactivity value than the others as a 'dominant' basin. SLN biopsy was performed only for the dominant basin. If two basins showed similar radioactivity, biopsy was conducted for both.

Results: One hundred and seven patients underwent preoperative lymphoscintigraphy, and 29 dual (popliteal, inguinal) drainages were found. Of the 29 melanomas with dual drainage, seven showed similar radioactivity on both basins, 22 showed inguinal as the dominant basin and no melanoma had a dominant popliteal basin. Among the seven patients who underwent dual-basin biopsy, six had negative results, all of which experienced no recurrence during follow-up. Among the 22 patients who underwent biopsy of the dominant inguinal basin, 19 had negative results. During the follow-up, these patients showed six recurrences, but none of them experienced popliteal recurrence. All three patients who had tumor-positive inguinal node experienced recurrence in the popliteal basin as well as at other sites.

Conclusion: For melanomas showing dual-basin drainage on lymphoscintigraphy, performing SLN biopsy only on the dominant (inguinal) basin is reliable when the dominant basin shows negative results. However, considering the recurrence pattern of our data, a tumor-positive dominant basin should prompts an SLN biopsy of the other basin.

Keywords: Melanoma, Lower extremity, Sentinel lymph node biopsy, Lymphoscintigraphy, Popliteal

\section{INTRODUCTION}

Melanoma is a skin cancer that rarely occurs in Asian countries [1]. According to data from the Korea Central Cancer Registry, it accounts for $0.3 \%$ of total cancer incidence in Korea as of 2014 . The current standard of care for melanomas that are clinically node negative is wide local excision of the primary tumor and sentinel

Received: Sep 28, 2017 Accepted: Dec 15, 2017

Correspondence to: Jung-Han Kim

Department of Surgery, Samsung Medical Center, Sungkyunkwan

University School of Medicine, 81 Irwon-ro, Gangnam-gu, Seoul 06351,

Korea

Tel: +82-2-3410-0286, Fax: +82-2-3410-6982

E-mail: jinnee.kim@samsung.com

Copyright ( $($ Korean Society of Surgical Oncology

This is an Open Access article distributed under the terms of the Creative Commons Attribution Non-Commercial License (http://creativecommons.org/licenses/by-nc/4.0) which permits unrestricted non-commercial use, distribution, and reproduction in any medium, provided the original work is properly cited. lymph node (SLN) biopsy for regional nodal staging [2]. Lymphoscintigraphy is performed prior to surgery to identify the location and number of SLNs. Sometimes, SLNs are detected in unexpected areas, such as the internal mammary nodes in breast cancer and the popliteal lymph nodes in distal lower extremity melanoma cases. Almost all melanomas with popliteal drainage show concurrent drainage to inguinal basin [3]. Dual-basin drainage is rare in melanoma patients; as a result, literature concerning popliteal SLNs and their management is limited [4]. This study aimed to analyze clinical outcomes and feasibility of SLN biopsies in lower extremity melanomas with dual-basin drainage on lymphoscintigraphy.

\section{METHODS}

Patients

This was a retrospective analysis of 107 patients with lower extremity melanoma who underwent lymphoscintigraphy and SLN 
biopsy in a single center between January 2006 to December 2015. Patients with confirmed nodal metastasis before surgery and patients with melanoma in situ who did not require an SLN biopsy were excluded.

\section{Methods}

All patients underwent subcutaneous injection of technetium 99-phytate $2 \mathrm{mCi}$ around the primary tumor on the morning of the surgery, followed by 30-minute continuous imaging and delayed whole body imaging (Fig. 1). A handheld gamma probe (NEO2000, Neoprobe Co., Dublin, OH, USA) was used to detect SLNs during the operation. In the case of dual-basin (inguinal and popliteal) drainage, we regarded the basin showing two times or greater radioactivity value than the other as the 'dominant' basin, and SLN biopsy was performed only in the dominant basin. If the two basins showed similar radioactivity, biopsy was performed for both. Complete lymph node dissection (CLND) was performed for all tumor-positive basins. The patients visited the outpatient

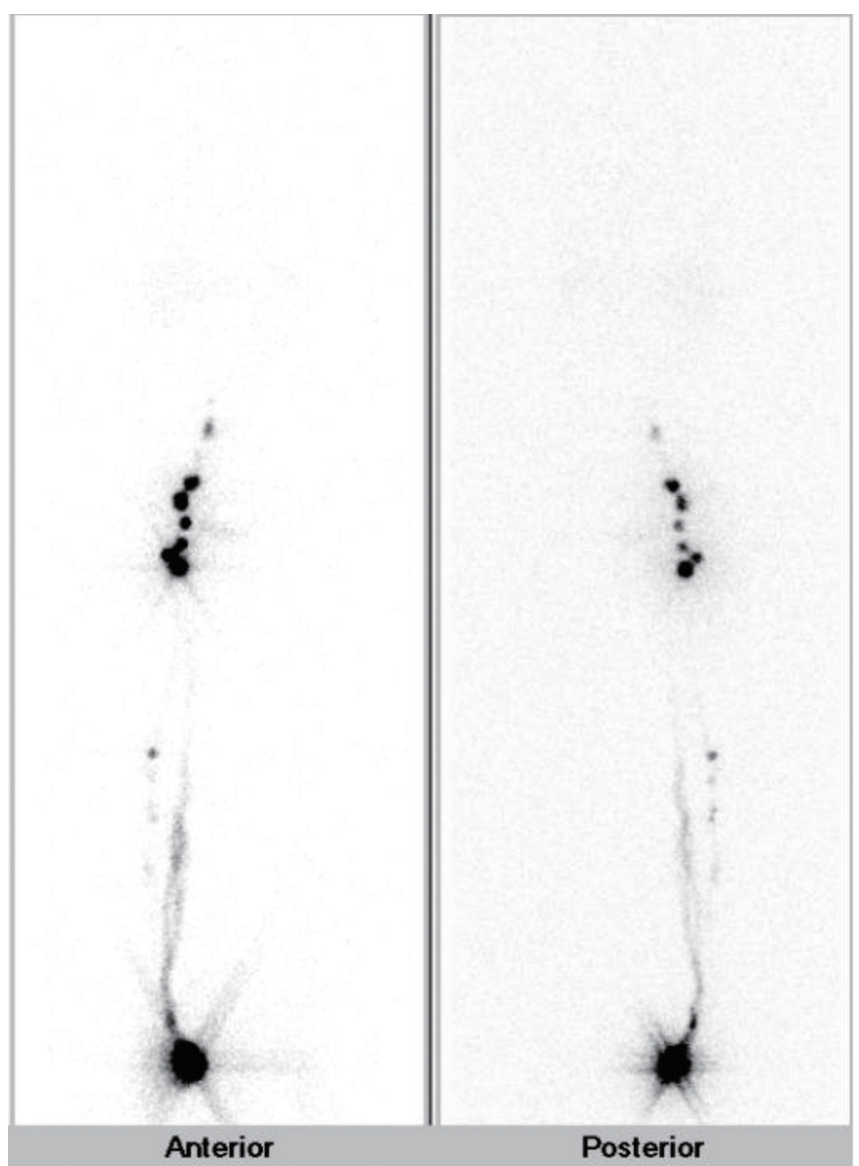

Fig. 1. Lymphoscintigraphy of a patient with primary melanoma on right heel, showing multifocal radiouptake in the right lower leg, popliteal, and ilioinguinal areas. clinic every 6 months for the first 5 years and annually thereafter. Physical examination and ultrasonography around the inguinal region and primary tumor site were performed. Chest and abdominopelvic computed tomography scans were also performed every 6 months or 1 year to detect recurrence during the follow-up period. We used SPSS for Windows (ver. 22.0, IBM Corp., Armonk, NY, USA) for statistical evaluation. Student t-test and Pearson chisquare analysis were used to compare patient characteristics, and survival rates were calculated using the Kaplan-Meier method. A P-value $<0.05$ was considered statistically significant.

\section{RESULTS}

\section{Patient characteristics}

The mean age of the patients at the time of operation was 60.4 years (range, 29-88 years). The mean tumor depth was $3.41 \mathrm{~mm}$

Table 1. Clinicopathologic characteristics of the positive and negative popliteal basin drainage groups

\begin{tabular}{|c|c|c|c|c|}
\hline Characteristic & $\begin{array}{l}\text { All patients } \\
(n=107)\end{array}$ & $\begin{array}{c}\text { Popliteal } \\
\text { drainage }(+) \\
(n=30)\end{array}$ & $\begin{array}{c}\text { Popliteal } \\
\text { drainage }(-) \\
(n=77)\end{array}$ & P-value \\
\hline Age (yr) & $60.4 \pm 13.2$ & $61.1 \pm 12.9$ & $60.1 \pm 13.3$ & 0.731 \\
\hline Sex & & & & 0.244 \\
\hline Male & $51(48)$ & $17(57)$ & $34(44)$ & \\
\hline Female & $56(52)$ & $13(43)$ & $43(56)$ & \\
\hline Tumor depth (mm) & $3.41 \pm 3.6$ & $4.08 \pm 4.1$ & $3.14 \pm 3.3$ & 0.227 \\
\hline Tumor depth $>1 \mathrm{~mm}$ & $80(75)$ & $23(77)$ & $57(74)$ & 0.778 \\
\hline Tumor depth $>4 \mathrm{~mm}$ & $34(32)$ & $13(43)$ & $21(27)$ & 0.109 \\
\hline Tumor location & & & & $<0.001$ \\
\hline $\begin{array}{l}\text { Anterior aspect of the } \\
\text { sole, toe }\end{array}$ & $54(51)$ & $4(13)$ & $50(65)$ & \\
\hline $\begin{array}{l}\text { Posterior aspect of the } \\
\text { sole, heel, calf }\end{array}$ & 53 (49) & $26(87)$ & $27(35)$ & \\
\hline Tumor site & & & & 0.453 \\
\hline Right & $58(54)$ & $18(60)$ & $40(52)$ & \\
\hline Left & $49(46)$ & $12(40)$ & $37(48)$ & \\
\hline Ulceration (+) & & & & 0.098 \\
\hline Absent & $49(46)$ & $11(37)$ & $38(49)$ & \\
\hline Present & $16(15)$ & $8(26)$ & $8(11)$ & \\
\hline Not available & $42(39)$ & $11(37)$ & $31(40)$ & \\
\hline $\begin{array}{l}\text { Lymphovascular inva- } \\
\text { sion (+) }\end{array}$ & & & & 0.328 \\
\hline Absent & $65(61)$ & $21(70)$ & $44(57)$ & \\
\hline Present & $3(3)$ & 0 & $3(4)$ & \\
\hline Not available & $39(36)$ & $9(30)$ & $30(39)$ & \\
\hline Retrieved SLN No. & $2.74 \pm 1.8$ & $2.73 \pm 2.0$ & $2.75 \pm 1.7$ & 0.961 \\
\hline
\end{tabular}

Values are presented as mean \pm standard deviation or number (\%). SLN, sentinel lymph node. 
(range, $0.1-15 \mathrm{~mm}$ ), and the mean number of harvested SLNs per patient was 2.74 (range, $0-11$ ). Drainage to the popliteal basin was observed in 30 (28\%) patients on lymphoscintigraphy. We compared the clinicopathological characteristics of the patients with popliteal drainage and the patients without popliteal drainage (Table 1). Most of the characteristics were not significantly different, but the majority of popliteal drainage group tumors were significantly more likely to be located on the posterior aspect of the sole $(87 \%)$ than the anterior aspect of the sole $(13 \%)(\mathrm{P}<0.001)$.

\section{Surgical management}

Among 30 patients with popliteal drainage, 29 showed concurrent inguinal drainage and one patient had only popliteal drainage. Of the 29 patients with dual-basin drainage, seven showed similar gamma radioactivity in both basins and so underwent biopsy for both basins. In the remaining 22 patients, the inguinal basin was diagnosed as dominant, and only inguinal biopsy was performed. No melanoma presented with a popliteal-dominant basin.

\section{Recurrence after sentinel node biopsy}

We analyzed patient outcome after SLN biopsy to determine the appropriateness of our surgical strategy when uptake on lymphoscintigraphy was in both lymph node basins. At an average fol- low-up period of 41 months, 36 (33.6\%) patients experienced recurrence. Two (1.9\%) patients showed local recurrence in the lower leg, seven (6.5\%) patients showed in-transit recurrence, one (0.9\%) patient showed recurrence in the non-operated lymph node basin, five (4.7\%) showed recurrence in the operated lymph node basin, $10(9.3 \%)$ showed systemic recurrence, and 11 (10.3\%) showed combined recurrences. Of the 77 patients with inguinal only drainage, 25 (32.4\%) experienced recurrence; of the 29 patients with dual-basin drainage, 10 (34.4\%) experienced recurrence. Three-year disease-free survival (DFS) and overall survival (OS) were estimated between the group with popliteal drainage and group without popliteal drainage. Patients with popliteal drainage had worse OS $(\mathrm{P}<0.001)$ but not DFS $(\mathrm{P}=0.242)$.

Of the seven patients who underwent dual-basin biopsy, six had tumor-negative SLNs on both basins, none of whom experienced recurrence during follow-up. One patient had tumor-positive SLNs in both basins and underwent CLND. However, the patient experienced systemic recurrence 22 months after the surgery. Of the 22 patients who underwent SLN biopsy of the dominant-inguinal basin, 19 had a tumor-negative result. During follow-up, six of these patients experienced recurrence (three showed systemic recurrence, two in-transit recurrence, and one inguinal lymph node recurrence), but none of them experienced recurrence in the

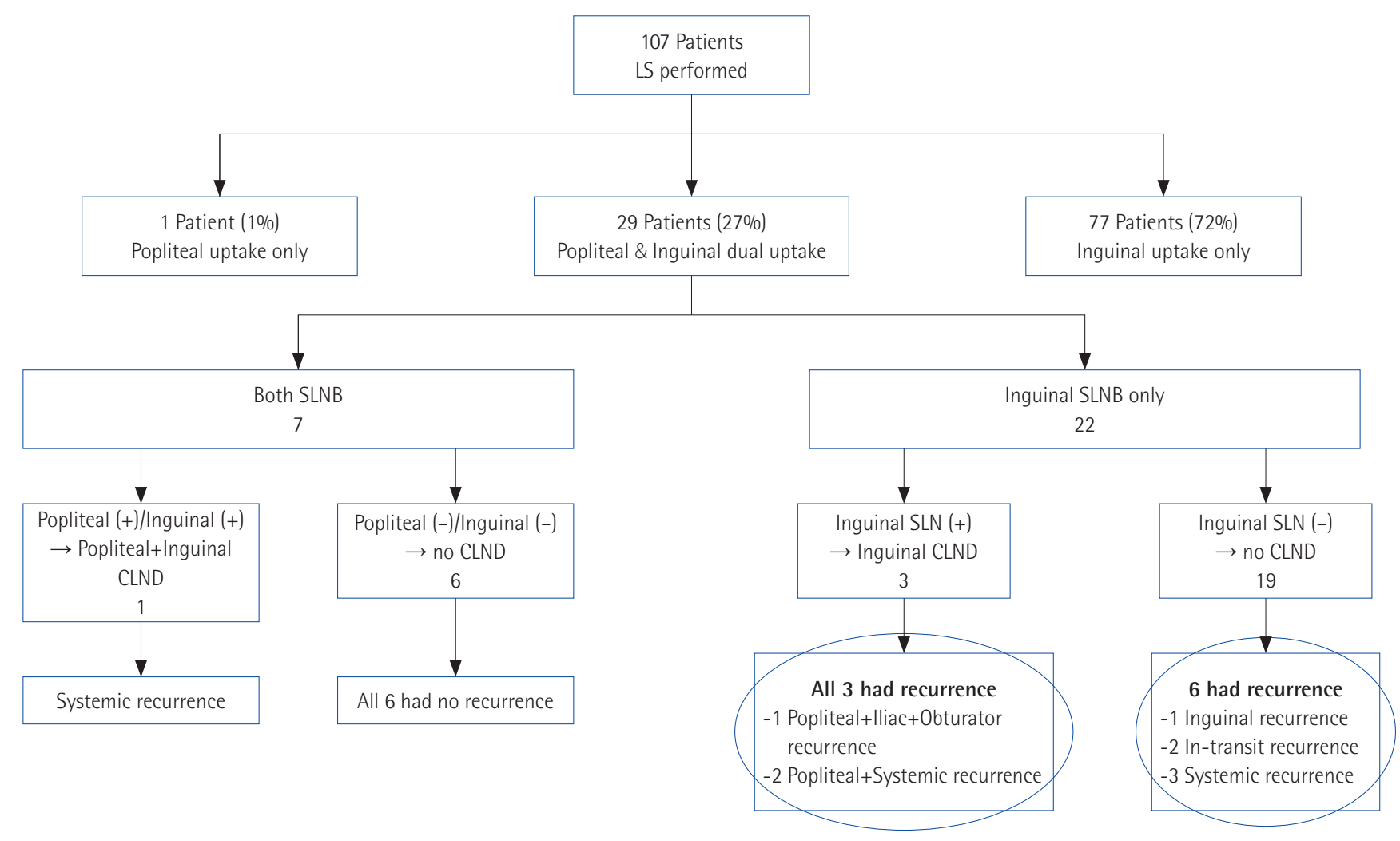

Fig. 2. Flow chart of study findings of patients. LS, lymphoscintigraphy; SLNB, sentinel node biopsy; CLND, complete lymph node dissection. 
popliteal basin. Three patients with tumor-positive inguinal nodes underwent CLND, but all three patients experienced recurrence in the popliteal space as well as at another site (Fig. 2).

\section{DISCUSSION}

SLN biopsy in melanomas has been widely used as a standard treatment with primary tumor excision, because it can be used as a regional nodal staging tool for clinically occult nodal metastasis [5]. The conventional SLN basin of lower extremity melanoma is the inguinal basin. Popliteal drainage is an uncommon event and popliteal node metastasis cases are rare. Although previous studies recommend performing biopsies on all of the basins that showed uptake on preoperative lymphoscintigraphy, some surgeons do not perform popliteal node biopsy for several reasons. First, surgical experience with the popliteal area is limited. Second, operation on the popliteal space has potential risk of complications, such as permanent damage to the tibial and peroneal nerves. Finally, biopsy of the popliteal space requires position change of the patient during the surgery, which results in a longer operation time $[4,6,7]$.

Previous studies about lymphatic anatomy showed that lymphatic drainage from the medial aspect of the foot and ankle follows the greater saphenous vein and drains to the inguinal area, while lymphatics from the posterolateral aspect of the heel, lateral foot, and ankle follow the lesser saphenous vein and drain to the popliteal area [6,8]. Many studies showed that this is also true for lymphatic drainage in melanoma cases. Although the majority of our patients with popliteal drainage (87\%) also had their primary tumor located on the posterior aspect of the foot, four (13\%) had a primary tumor located on the first toe (medial aspect of the foot). Kretschmer et al. [8] and Thompson et al. [9] also suggested that any skin site below the knee can drain to the popliteal fossa.

In our study, popliteal drainage were found in $28 \%$ of cases, which is significantly more than that found in previous studies (1\%-20\%) [9-12]. Uren et al. [13] reported that, after changing the imaging protocol, the rate of popliteal drainage increased from $2.3 \%$ to $16 \%$. We assume that the difference in the protocol and the method for performing lymphoscintigraphy and misinterpretation of images could lead to variability in the rate of popliteal drainage, as in our study.

Almost all melanomas with popliteal drainage also had concurrent inguinal drainage. Several studies have explained at least three distinct patterns of lymphatic drainage to the popliteal basin. First, the popliteal basin is an in-transit node, so the single lymphatic channels drain serially to the popliteal area and continue to the inguinal region. In the second pattern, both popliteal and inguinal basins are first-order sentinel node, so two or more lymphatic channels drain into each basin. In the third pattern, the serial and parallel drainage are mixed, so the inguinal basin can be a first- or second-order basin [6,7]. However, the results of lymphoscintigraphy do not always correspond to one of the three lymphatic drainage patterns. If we classify the lymphatic drainage patterns according to the amount of radioactivity measured by a gamma probe during the operation, the amount measured in the popliteal area would be remarkably high in cases of serial drainage. In such cases, the popliteal area can be regarded as an SLN and a biopsy can be performed in only the popliteal area. In cases of parallel or mixed drainage, the two basins will show similar radioactivity. We assumed that metastasis would occur first in the basin that has higher lymphatic flow. Therefore, if we can detect the basin with higher lymphatic flow before surgery, we can omit biopsy of the minor basin, reducing the operation time and complication rate. We divided dual-basin drainage cases into two groups. If the radioactivity value of the greater basin showed two times or greater radioactivity value than the other, we regarded it as the 'dominant' basin and performed SLN biopsy only for that basin. If the radioactivity value of the greater basin was less than twice the value of the other basin, we regarded both basins as 'dominant', and SLN biopsy was performed for both.

We analyzed the recurrence pattern of patients after the surgery to determine if our strategy is feasible. Of the 22 patients with an inguinal dominant basin, 19 showed tumor-negative SLN. They had no recurrence at the popliteal area during the follow-up period. It is rational to assume that there would be no metastasis to the lymph node of the minor basin (in our case, the popliteal region) if there is no metastasis in the dominant basin. In contrast, all three patients with metastasis in the dominant inguinal basin had recurrences also in the popliteal area just a few months after surgery. These results support the hypothesis that when the dominant basin has a tumor-positive lymph node, additional biopsy of the SLN in the minor basin would help to more precisely determine the status of node metastasis.

A drawback of our surgical strategy is the risk of missed lymph node metastasis in the minor basin (popliteal) when there is no metastasis in the dominant basin (inguinal). Steen et al. [4] reported that 26\% (4 of 15) of patients with popliteal drainage had popliteal-only metastasis, while Bertolli et al. [14] reported that 4\% (1 of 27) had popliteal-only metastasis. However, these previous studies did not classify the lymphatic drainage pattern as we did and performed biopsy of all lymphatic areas that showed radioactive uptake, making it difficult to compare with the results of our study. There was no dominant popliteal basin in our study, which suggests that we were not able to visualize such metastasis.

It is still under debate whether lymph node dissection should be 
continued after detection of tumor-positive SLN. Recently, the DeCOG-SLT trial has been published on the analysis of a prospective randomized controlled study of 483 patients with melanoma who had tumor-positive SLN. The results showed no difference in survival in patients treated with CLND compared with observation only [15]. They recommended that CLND not be performed in patients with thin melanoma of $1 \mathrm{~mm}$ or smaller. This result suggests that SLN biopsy is helpful in predicting stage and survival rate, but CLND of a tumor-positive basin does not have a therapeutic benefit. If further prospective studies validate this hypothesis in all melanoma patients, it would be necessary to analyze approaches for finding an exact SLN basin, as in this study, in order to avoid unreasonable dual-basin surgery.

Our study has several limitations. First, it was a retrospective study, and the data were collected from a single center, possibly resulting in selection bias. Secondly, the number of patients was not large, and the follow-up period was relatively short. Third, there was no control group. Finally, it is difficult to generalize the present results because each medical institution has different imaging protocols and methods of lymphoscintigraphy. Additional methods and protocols to identify exact SLNs in melanoma patients showing dual-basin drainage should be discussed in further studies.

In summary, 27.1\% of lower extremity melanoma cases showed dual-basin drainage on lymphoscintigraphy. Popliteal-only drainage was very rare, and there were no cases with popliteal-dominant basin on our gamma probe analysis. Performing SLN biopsy only on the dominant (inguinal) basin is reliable when that basin shows negative results. However, if a dominant basin is tumor positive, an additional SLN biopsy of the other basin should be considered to more precisely determine the status of node metastasis.

\section{CONFLICT OF INTEREST}

No potential conflict of interest relevant to this article was reported.

\section{REFERENCES}

1. Simard EP, Ward EM, Siegel R, Jemal A. Cancers with increasing incidence trends in the United States: 1999 through 2008. CA Cancer J Clin 2012;62:118-28.

2. Madu MF, Wouters MW, van Akkooi AC. Sentinel node biopsy in melanoma: Current controversies addressed. Eur J Surg Oncol 2017;43:517-33.

3. Fitzgerald TL, Gronet EM, Atluri P, Zervos EE, Wong JH. Patterns of node mapping differ for axial and extremity primary cutaneous melanoma: a case for a more selective use of pre-operative imaging. Surgeon 2016;14:190-5.

4. Steen ST, Kargozaran H, Moran CJ, Shin-Sim M, Morton DL, Faries MB. Management of popliteal sentinel nodes in melanoma. J Am Coll Surg 2011;213:180-6.

5. Gershenwald JE, Thompson W, Mansfield PF, Lee JE, Colome MI, Tseng CH, et al. Multi-institutional melanoma lymphatic mapping experience: the prognostic value of sentinel lymph node status in 612 stage I or II melanoma patients. J Clin Oncol 1999;17:976-83.

6. Menes TS, Schachter J, Steinmetz AP, Hardoff R, Gutman H. Lymphatic drainage to the popliteal basin in distal lower extremity malignant melanoma. Arch Surg 2004;139:1002-6.

7. Morcos B, Al-Ahmad F. Metastasis to the popliteal lymph nodes in lower extremity melanoma and their management. Surg Oncol 2011;20:e119-22.

8. Kretschmer L, Sahlmann CO, Bardzik P, Thoms KM, Bertsch HP, Meller J. The popliteal fossa: a problem zone for sentinel lymphonodectomy. J Dtsch Dermatol Ges 2011;9:123-7.

9. Thompson JF, Hunt JA, Culjak G, Uren RF, Howman-Giles R, Harman CR. Popliteal lymph node metastasis from primary cutaneous melanoma. Eur J Surg Oncol 2000;26:172-6.

10. Lieber KA, Standiford SB, Kuvshinoff BW, Ota DM. Surgical management of aberrant sentinel lymph node drainage in cutaneous melanoma. Surgery 1998;124:757-61.

11. Sumner WE 3rd, Ross MI, Mansfield PF, Lee JE, Prieto VG, Schacherer CW, et al. Implications of lymphatic drainage to unusual sentinel lymph node sites in patients with primary cutaneous melanoma. Cancer 2002;95:354-60.

12. McMasters KM, Chao C, Wong SL, Wrightson WR, Ross MI, Reintgen DS, et al. Interval sentinel lymph nodes in melanoma. Arch Surg 2002;137:543-7.

13. Uren RF, Howman-Giles RB, Thompson JF. Failure to detect drainage to the popliteal and epitrochlear lymph nodes on cutaneous lymphoscintigraphy in melanoma patients. J Nucl Med 1998; 39:2195.

14. Bertolli E, Bevilacqua JL, Molina AS, de Macedo MP, Pinto CA, Duprat Neto JP. Popliteal sentinel lymph node involvement in melanoma patients. J Surg Oncol 2015;112:179-82.

15. Leiter U, Stadler R, Mauch C, Hohenberger W, Brockmeyer N, Berking C, et al. Complete lymph node dissection versus no dissection in patients with sentinel lymph node biopsy positive melanoma (DeCOG-SLT): a multicentre, randomised, phase 3 trial. Lancet Oncol 2016;17:757-67. 